\title{
Hábitos alimentares de crianças menores de 1 ano amamentadas e não-amamentadas
}

\author{
Feeding habits of breastfed and non-breastfed children up to 1 year old \\ Graciete O. Vieira', Luciana R. Silva ${ }^{2}$, Tatiana de O. Vieira33, \\ João Aprígio G. Almeida4, Vilma A. Cabral 5
}

\section{Resumo}

Objetivos: Descrever os hábitos alimentares de crianças amamentadas e não-amamentadas através da análise dos alimentos consumidos pelas crianças menores de 1 ano de idade na cidade de Feira de Santana, BA, no ano de 2001.

Métodos: Foi realizado um estudo transversal, no qual foram aplicados questionários às mães das crianças menores de 1 ano no dia nacional de vacinação, presentes nas $44(71 \%)$ unidades de vacinação, selecionadas por estratificação simples. Foram avaliadas 2.319 crianças, representando $24,3 \%$ da população estimada. As medidas de associação calculadas foram: razão de prevalência, com intervalo de confiança a $95 \%$, e de significância estatística, através do teste do quiquadrado com respectivo valor $\mathrm{p}$.

Resultados: Nas crianças com idade igual ou inferior a 4 meses e não-amamentadas, foi observada uma chance 8,2 e 6,7 vezes maior de a criança ser alimentada, respectivamente, com a refeição da família (IC $95 \% 3,23-20,66$ ) e com papas de legumes (IC 95\% 3,84-11,78). Na mesma faixa etária, as prevalências de crianças não-amamentadas que receberam água, chás, sucos e papa de frutas foram significativamente maiores $(70,7 \%$ versus $19,7 \%, 63,3 \%$ versus $26,7 \%, 33,3 \%$ versus $4,9 \%$ e $14,4 \%$ versus $1,4 \%$, respectivamente).

Conclusões: As crianças amamentadas, quando comparadas com as não-amamentadas, apresentaram melhores hábitos no que se refere à época de introdução dos alimentos complementares.

J Pediatr (Rio J). 2004;80(5):411-6: Aleitamento materno, alimentação, comportamento alimentar, alimentação mista.

1. Doutoranda em Medicina e Saúde, Curso de Pós-Graduação, Universidade Federal da Bahia (UFBA). Professora assistente, Universidade Estadual de Feira de Santana (UEFS), Feira de Santana, BA.

2. Professora titular de Pediatria e Chefe do Serviço de Gastroenterologia e Hepatologia Pediátrica, UFBA, Salvador, BA.

3. Bolsista de Iniciação Científica, Fundo de Amparo à Pesquisa no Estado da Bahia (FAPESB). Monitora de SPSS do Curso de Pós-Graduação em Medicina e Saúde, UFBA. Acadêmica de Medicina, Escola Bahiana de Medicina e Saúde Pública (EBMSP), Salvador, BA.

4. Doutor em Saúde Pública, Instituto Fernandes Figueira/Fundação Oswaldo Cruz (IFF/FIOCRUZ). Coordenador do Centro de Referência Nacional para Bancos de Leite Humano.

5. Mestre em Saúde Coletiva, UFBA. Oficial de Projeto UNICEF, Bahia e Sergipe.

Artigo submetido em 28.10.03, aceito em 23.06.04.

Como citar este artigo: Vieira GO, Silva LR, Vieira TO, Almeida JAG, Cabral VA. Hábitos alimentares de crianças menores de 1 ano amamentadas e não-amamentadas. J Pediatr (Rio J). 2004;80:411-6.

\section{Abstract}

Objective: To describe the eating habits of breastfed and nonbreastfed children through the analysis of food intake by children younger than one year of age in Feira de Santana in 2001.

Methods: Cross-sectional study. The mothers of children younger than one year answered a questionnaire during the national vaccination day in $44(71 \%)$ vaccination units selected by simple stratification. 2,319 children were evaluated, representing $24.3 \%$ of the estimated population. Prevalence ratio and $95 \%$ confidence interval were calculated. Statistical significance was determined using the chisquare test.

Results: Non-breastfed children aged four months or younger were 8.2 and 6.7 times more likely to receive the family food ( $95 \%$ CI: 3.23-20.66) or vegetable soup ( $95 \%$ CI: $3.84-11.78$ ), respectively. In the same age group, the prevalence of non-breastfed children receiving water, juice, and fruit was significantly higher in relation to breastfed children $(70.7 \%$ vs $19.7 \%, 63.3 \%$ vs $26.7 \%, 33.3 \%$ vs $4.9 \%$ and $14.4 \%$ vs $1.4 \%$, respectively).

Conclusion: Breastfed children had healthier habits than nonbreastfed children in terms of the introduction of complementary foods.

J Pediatr (Rio J). 2004;80(5):411-6: Breast feeding, feed, feeding behavior, mixed feeding.

\section{Introdução}

O leite humano oferece os nutrientes que a criança necessita para iniciar uma vida saudável e representa o alimento essencial para o lactente até o sexto mês de vida, como alimento exclusivo; a partir de então, deve ser complementado com outras fontes nutricionais até pelo menos 2 anos de idade ${ }^{1,2}$.

Documentos anteriores da Organização Mundial de Saúde (OMS) recomendavam o aleitamento exclusivo por 4-6 meses de vida ${ }^{3}$. Baseados em evidências científicas dos benefícios do aleitamento exclusivo, muitos países, dentre eles o Brasil, assumiram oficialmente a recomendação de alimentos complementares aos 6 meses de idade ${ }^{4,5}$. Atualmente, a OMS e a política nacional coincidem na recomendação da amamentação exclusiva nos 6 primeiros meses de vida ${ }^{1,5}$. 
Na busca de um consenso sobre a duração ótima do aleitamento materno exclusivo, a OMS realizou uma revisão sistemática das pesquisas publicadas e concluiu que nenhuma delas mostrou déficit no ganho de peso e altura para as crianças alimentadas exclusivamente com leite materno no primeiro semestre de $v^{1} a^{1}$. O principal argumento contra a introdução dos alimentos complementares antes do sexto mês de vida foi o aumento do risco de episódios de infecções gastrintestinais ${ }^{1,4,6,7}$.

Além do aumento da morbimortalidade infantil, são inúmeras as desvantagens da introdução precoce dos alimentos complementares, entre as quais ressaltam-se a interferência na absorção de nutrientes, como o ferro e o zinco ${ }^{4}$, o aumento do risco de alergia alimentar ${ }^{6}$, e a maior ocorrência de doenças crônico-degenerativas na idade adulta ${ }^{8}$.

Ademais, com a introdução dos alimentos complementares antes dos 6 meses de idade, a criança passa a ingerir menos leite humano, com a conseqüente diminuição da produção de leite pela mãe nutriz ${ }^{4}$, redução na duração do aleitamento materno, diminuição da eficácia da lactação como meio contraceptivo ${ }^{1}$ e interferência no comportamento alimentar do bebê ${ }^{8}$. Mesmo em crianças não-amamentadas, a recomendação habitual para a introdução dos alimentos sólidos é após os 4 meses de vida9 .

Entretanto, a nutrição de uma população não depende só do acesso desta a uma alimentação adequada, mas, sobretudo, da educação e cultura da população. A educação alimentar inicia-se muito precocemente, nos primeiros meses de vida, quando são construídos os alicerces dos hábitos alimentares ${ }^{8,10}$.

O comportamento alimentar da criança é determinado pela interação da criança com o alimento, pelo seu desenvolvimento anatomofisiológico e por fatores emocionais, psicológicos, socioeconômicos e culturais ${ }^{8}$. Entretanto, a influência mais marcante na formação dos hábitos alimentares é o produto da interação da criança com a própria mãe ou a pessoa mais ligada à sua alimentação ${ }^{9,11}$. É importante lembrar que os lactentes ingerem os alimentos que Ihes são oferecidos e do modo como são preparados.

A família oferece amplo campo de aprendizado social à criança. O ambiente doméstico, o estilo de vida dos pais, as relações interfamiliares podem ter grande influência na alimentação, nas preferências alimentares, e afetar o equilíbrio energético da alimentação pela disponibilidade e composição dos alimentos. Assim, a família poderá estabelecer o aprendizado de um hábito socialmente aceito ou inserir novos hábitos, contribuindo para a formação de um padrão de comportamento alimentar adequado ou não11.

O objetivo do presente estudo foi descrever os hábitos alimentares de crianças amamentadas e não-amamentadas segundo a introdução dos alimentos líquidos e semisólidos/sólidos no primeiro ano de vida. Foi também pesquisado o uso de chupetas conforme a presença ou ausência do aleitamento materno. Através do conhecimento de fatores associados aos hábitos alimentares, medidas educativas e preventivas podem ser propostas para a formação de um comportamento alimentar saudável e para a promoção da saúde da criança e do adulto.

\section{Métodos}

Este é um estudo de base populacional, do tipo transversal, aprovado pelo Comitê de Ética e Pesquisa da Universidade Federal da Bahia. A população do estudo foi constituída por crianças menores de 1 ano de idade, completo até o dia 25 de agosto de 2001, dia nacional de vacinação. As crianças eram procedentes da cidade de Feira de Santana, estavam presentes nos postos de vacinação selecionados e estavam acompanhadas de suas respectivas mães, as quais aceitaram responder ao questionário.

O desenho amostral foi estratificado casual simples. A vacinação ocorreu em 62 unidades (postos de saúde e escolas). Para assegurar a cobertura vacinal, a Secretaria de Saúde de Feira de Santana dividiu a cidade em quatro "comandos". Uma amostra casual simples foi retirada de cada estrato (comando). Foram, assim, sorteadas 44 (71\%) unidades dentre as 62 existentes. Foi calculada uma amostra correspondente a $20 \%$ (1.912) da população estimada para as crianças de até 11 meses e 29 dias (9.563). O questionário foi aplicado às $2.323(24,3 \%)$ mães das crianças.

A coleta de dados foi realizada por 104 universitários previamente treinados. O formulário para a coleta de dados foi construído com linguagem clara e objetiva, com respostas fechadas, mutuamente excludentes e, na grande maioria, com três alternativas: sim, não e não sei; o questionário foi aplicado sob forma de entrevista direta. Foi utilizado o recordatório das 24 horas que antecederam a coleta de dados para a pesquisa dos alimentos consumidos pela criança.

As duas principais variáveis estudadas foram: amamentação (independente principal) e hábito alimentar (dependente). O hábito alimentar foi analisado conforme o tipo de alimentos complementares introduzidos na dieta da criança e a idade desta na época da introdução. As variáveis externas pesquisadas, que poderiam interferir na associação principal, foram: idade da criança em dias, sexo, peso no nascimento e hábito de sugar chupetas. As características maternas inquiridas foram: idade, paridade, escolaridade, trabalho fora do lar e renda familiar.

Por alimento complementar entendeu-se qualquer alimento nutritivo líquido ou semi-sólido/sólido oferecido à criança amamentada ${ }^{4}$. Os alimentos líquidos questionados foram: água, chás, sucos; foram considerados como alimentos semi-sólidos frutas e legumes sob a forma de papas. A refeição da família foi definida como alimento sólido preparado para a alimentação habitual dos adultos. Estavam em aleitamento exclusivo as mães que afirmavam dar somente leite do peito aos seus filhos.

Para a análise dos dados, foram calculadas medidas de associação (razão de prevalência), com intervalo de confiança a $95 \%$, e de significância estatística, através do 
teste do qui-quadrado com respectivo valor p. O programa estatístico utilizado foi o Statistical Package for Social Science (SPSS), versão 10.0 .

\section{Resultados}

Foram entrevistadas 2.323 mães, sendo quatro questionários desprezados por terem dados incompletos. No dia da coleta de dados, 69,2\% (1.603) dos menores de 1 ano estavam mamando. A prevalência de aleitamento materno exclusivo nos menores ou iguais a 4 e 6 meses, foi, respectivamente, de 48,3 e 38,5\%.

Quanto às características maternas, 58,8\% estavam na faixa etária entre 20-29 anos, 46,2\% eram primíparas, e $23,3 \%$ trabalhavam fora do domicílio. Das 1.640 mães que sabiam a renda familiar, 49,6\% informaram ser a mesma entre um e dois salários mínimos. Em relação à escolaridade, $37,5 \%$ tinham nível de instrução maior que o ensino básico fundamental. Dentre as 2.319 crianças estudadas, $52,8 \%$ eram menores ou iguais a 6 meses, $7,3 \%$ nasceram com peso inferior a 2.500 gramas, e 49,9\% eram do sexo masculino.

No primeiro mês de vida, as crianças amamentadas já bebiam água $(7,3 \%)$, chás $(23,2 \%)$ e sucos $(2,7 \%)$ (Figura 1); nas crianças não-amamentadas, o consumo de água, chás e sucos foi significativamente maior, sendo, respectivamente, de $30 \%(p=0,01), 50 \%(p=0,05)$ e $30 \%(p=$ $0,000)$ em comparação às amamentadas.

O consumo de alimentos semi-sólidos/sólidos pelas crianças amamentadas no primeiro mês de vida foi de $0,7 \%$ para as papas de frutas e refeição da família e de $2 \%$ para as papas de legumes. Não foi observado um aumento importante de ingestão desses alimentos nos primeiros 3 meses de vida (Figura 2). Nos lactentes não-amamentados, as papas de frutas, papas de legumes e refeições da família foram significativamente mais consumidas no primeiro mês de vida, com respectivas cifras de $10 \%(p=0,01), 20 \%$ ( $p$ $=0,02)$ e $10 \%(p=0,01)$.

As crianças menores ou iguais a 4 meses não-amamentadas, comparadas com as amamentadas, apresentaram prevalências significativamente maiores quanto ao uso de água, chás, sucos e papas de frutas (Tabela 1 ). Na mesma faixa de idade, os lactentes não-amamentados apresentaram chances significativamente maiores em relação ao consumo de papas de legumes e refeição da família de modo precoce (Tabela 1). Quando se comparou o uso de chupeta entre os dois grupos, observaram-se também significativas diferenças, com maiores prevalências dentre as crianças não-amamentadas (Tabela 1).

Não foram observadas diferenças estatisticamente significativas quando se avaliou a introdução de refeição da família na alimentação das crianças menores ou iguais a 4 meses, segundo as co-variáveis maternas pesquisadas: idade menor que 20 anos $(p=0,61)$, escolaridade menor que o ensino básico fundamental $(p=0,19)$, trabalho fora do $\operatorname{lar}(p=0,20)$, renda familiar menor que dois salários mínimos $(p=0,29)$.
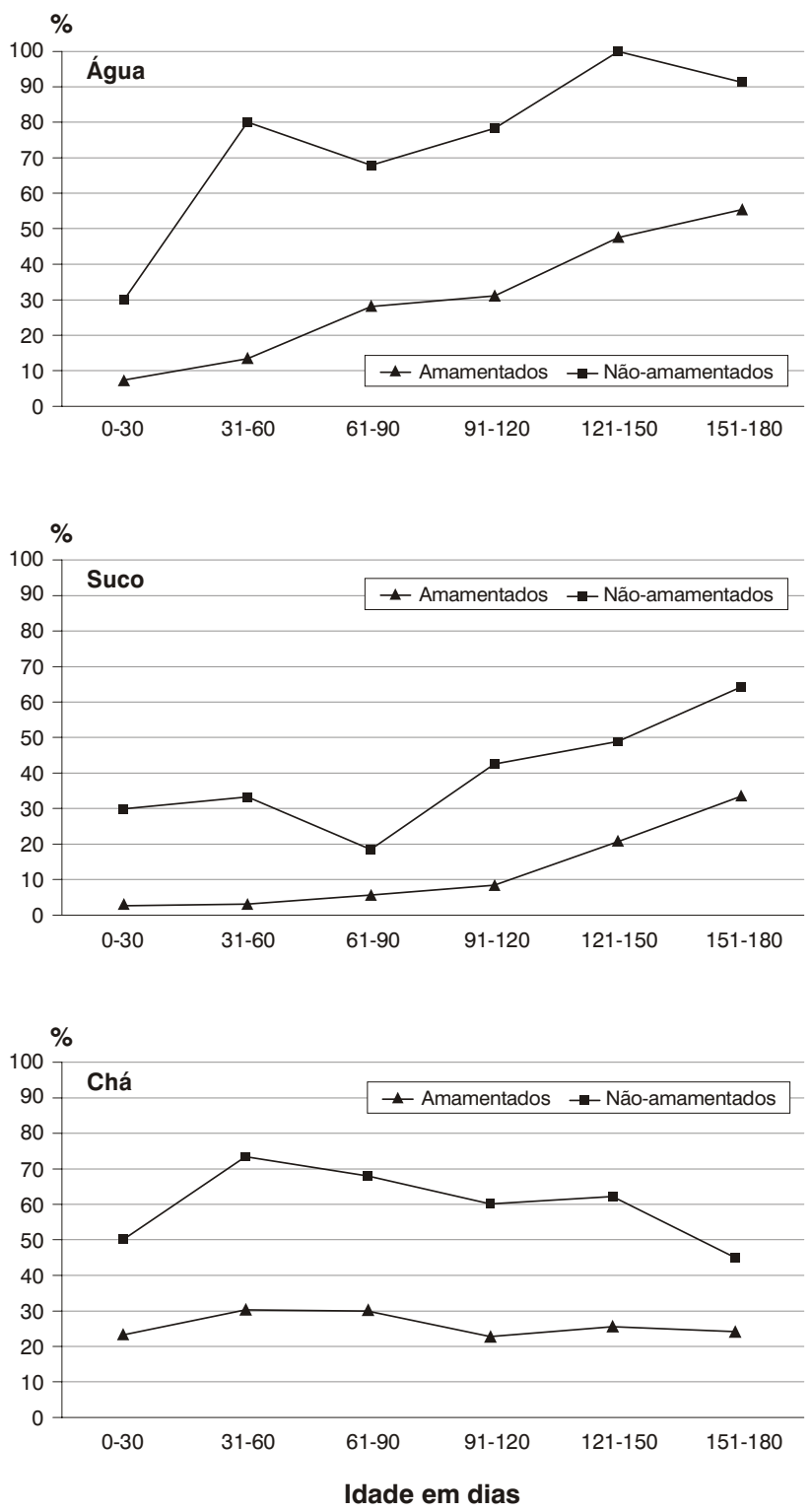

Figura 1 - Prevalência de crianças menores ou iguais a 6 meses que receberam água, suco e chá nas 24 horas que antecederam a pesquisa

\section{Discussão}

Nos últimos anos, acumularam-se evidências científicas que fundamentam a importância da amamentação exclusiva nos primeiros 6 meses de vida e a manutenção do aleitamento materno até pelo menos 2 anos de idade ${ }^{1,2,5}$.

Embora o aleitamento materno na cidade de Feira de Santana tenha revelado maiores prevalências quando comparado com os diversos estudos nacionais 7,12 , além de melhores hábitos alimentares das crianças amamentadas quando comparadas àquelas não-amamentadas, ficou demonstrado, no entanto, um alto consumo de alimentos líquidos, sobretudo os chás, desde o primeiro mês de vida. 

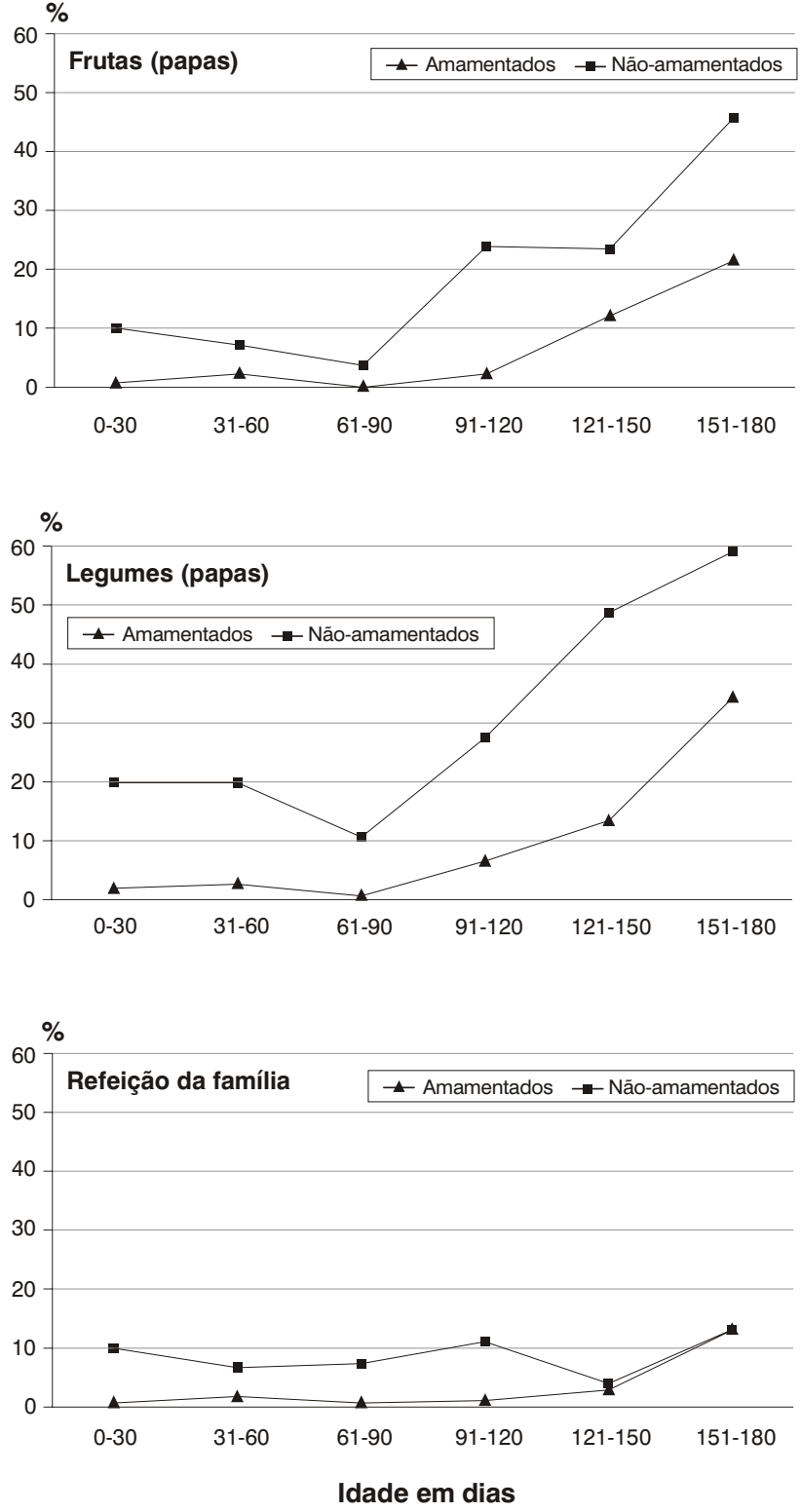

Figura 2 - Prevalência de crianças menores ou iguais a 6 meses que receberam frutas (papas), legumes (papas) e refeição da família nas 24 horas que antecederam a pesquisa

A equivalência de consumo de chás nos intervalos etários ao longo do primeiro ano, e o maior consumo quando comparado ao de água e sucos no primeiro mês de vida fortalecem a conviç̧ão de que, quando as mães oferecem chás aos seus filhos, o objetivo principal não é a nutrição infantil, mas sim a medicação, atendendo a fatores culturais, que reforçam o uso de chás como remédio.

Essa situação crítica quanto ao uso do chá foi demonstrada em outros estudos que constataram, também, a introdução precoce de chás e água na alimentação dos bebês ${ }^{13}$; as justificativas dadas pelas mães foram cólicas, gases e sede da criança ${ }^{14}$.
Tabela 1 - Consumo de água, suco, chá, papa de frutas e legumes, refeição da família e uso de chupeta conforme a presença ou ausência de aleitamento materno nas crianças menores ou iguais a 4 meses de idade

\begin{tabular}{|c|c|c|c|c|c|}
\hline \multirow[t]{3}{*}{ Variáveis } & \multicolumn{4}{|c|}{ Aleitamento materno } & \multirow{3}{*}{$\begin{array}{c}\text { RP } \\
\text { (IC } 95 \%)\end{array}$} \\
\hline & \multicolumn{2}{|c|}{ Não } & \multicolumn{2}{|c|}{ Sim } & \\
\hline & $\mathbf{n}$ & $\%$ & $\mathbf{n}$ & $\%$ & \\
\hline \multicolumn{6}{|l|}{ Água } \\
\hline Sim & 70 & 70,7 & 138 & 19,7 & 3,59 \\
\hline Não & 29 & 29,3 & 562 & 80,3 & $(2,95-4,36)$ \\
\hline \multicolumn{6}{|l|}{ Suco } \\
\hline Sim & 33 & 33,3 & 34 & 4,9 & 6,82 \\
\hline Não & 66 & 66,7 & 662 & 95,1 & $(4,44-10,49)$ \\
\hline \multicolumn{6}{|l|}{ Chá } \\
\hline Sim & 62 & 63,3 & 188 & 26,7 & 2,37 \\
\hline Não & 36 & 36,7 & 516 & 73,3 & $(1,95-2,88)$ \\
\hline \multicolumn{6}{|c|}{ Papa de frutas } \\
\hline Sim & 14 & 14,4 & 10 & 1,4 & 10,18 \\
\hline Não & 83 & 85,6 & 695 & 98,6 & $(4,65-22,27)$ \\
\hline \multicolumn{6}{|c|}{ Papa de legumes } \\
\hline Sim & 21 & 21,0 & 22 & 3,1 & 6,73 \\
\hline Não & 79 & 79,0 & 683 & 96,9 & $(3,84-11,78)$ \\
\hline \multicolumn{6}{|c|}{ Refeição família } \\
\hline Sim & 9 & 9,3 & 8 & 1,1 & 8,17 \\
\hline Não & 88 & 90,7 & 696 & 98,9 & $(3,23-20,66)$ \\
\hline \multicolumn{6}{|c|}{ Uso de chupeta } \\
\hline Sim & 82 & 82,0 & 383 & 53,9 & 1,52 \\
\hline Não & 18 & 18,0 & 328 & 46,1 & $(1,36-1,71)$ \\
\hline
\end{tabular}

RP (IC 95\%) = Razão de prevalência com intervalo de confiança a 95\%.

A influência rural observada na cidade de Feira de Santana pode ajudar a justificar os resultados encontrados, porque, sabidamente, as comunidades rurais têm hábitos mais tradicionais, e um grande número de mães inicia a amamentação e a mantém por mais tempo, porém introduzindo precocemente os alimentos complementares ${ }^{15}$.

Naturalmente, as crianças não-amamentadas ingeriram leite artificial, sobretudo nos primeiros meses de vida. Um dos problemas quanto à ingestão de proteína pelo bebê jovem diz respeito à maior permeabilidade da mucosa intestinal no período neonatal e nos 3 primeiros meses de vida, com conseqüente absorção de proteínas intactas, não digeridas, e resposta imunológica aos antígenos protéicos presentes nas fórmulas lácteas ${ }^{6}$. Essa característica, associada a mecanismos imunológicos, explica o grande número de crianças que desenvolvem intolerância ao leite de vaca, não só pelo alto poder antigênico da proteína heteróloga, como também pela precocidade do seu uso ${ }^{6}$. O leite de vaca é responsável por $20 \%$ das alergias alimentares ${ }^{4}$. Por esse motivo, as recomendações para a alimentação das crianças pequenas em muitos países incluem o adiamento da introdução de alguns alimentos por serem altamente alergênicos, dentre eles o leite de vaca; quando houver história familiar de alergia alimentar, sua introdução pode ser adiada para os 9-12 meses $^{4}$. 
Tem sido demonstrado que a utilização de outros alimentos na dieta da criança (além do leite de peito) eleva os riscos de morbimortalidade infantil por afecções associadas ao desmame precoce ${ }^{1,16-19}$. Estudo caso-controle avaliando o impacto do aleitamento na admissão hospitalar para tratamento da pneumonia demonstrou que a taxa de hospitalização por essa afecção foi significativamente maior em crianças que recebiam alimentos complementares antes dos 6 meses de vida, tanto para as crianças amamentadas, quanto para as alimentadas artificialmente ${ }^{18}$.

Arifeen, através de estudo de coorte, demonstrou que o aleitamento exclusivo conferiu uma forte proteção contra óbitos por diarréia e que o aleitamento predominante ou ausência de aleitamento estiveram associados a risco 2,2 vezes maior de morrer por outras causas de doenças infecciosas e a risco 3,9 vezes maior de morrer por infecções respiratórias e diarréia ${ }^{19}$.

$\mathrm{Na}$ atual pesquisa, foi evidenciado que, nos primeiros 3 meses de vida, o consumo de alimentos semi-sólidos/ sólidos pelas crianças amamentadas obteve baixas prevalências, com um aumento após o quarto mês. É possível que o término da licença maternidade e o retorno da mãe ao trabalho tenham contribuído para o início da alimentação complementar. Vale ressaltar que a recomendação da prática do aleitamento exclusivo por 4-6 meses de vida, presente ainda na grande maioria dos livros textos, baseados nas recomendações anteriores da $\mathrm{OMS}^{3}$, pode ter levado os próprios profissionais de saúde a orientar a introdução de alimentos complementares neste período, mesmo naqueles casos não caracterizados como hipogalactia, ou naquelas crianças com bom crescimento.

Ainda sobre os alimentos semi-sólidos/sólidos, foi constatado que crianças que não mamam consomem esse tipo de alimento inadequadamente desde o período neonatal, com diferenças estatisticamente significativas quando comparadas àquelas que mamam. As crianças estudadas, embora já consumissem alimentos complementares, apresentaram melhores hábitos de nutrição quando comparadas aos dados da Pesquisa Nacional de Demografia e Saúde, em que $52,9 \%$ das crianças não-amamentadas e $6,2 \%$ das amamentadas já comiam grão de aveia e/ou cereal no primeiro mês de vida ${ }^{20}$.

Além de as crianças não estarem fisiologicamente preparadas para digerir alimentos sólidos antes do sexto mês de vida, existe também uma imaturidade neurológica para deglutir alimentos não-líquidos, evidenciada pela presença do reflexo de protrusão da língua, através do qual a criança empurra os objetos que são aproximados dos seus lábios por isso, normalmente, rejeitam a alimentação oferecida de colher $^{3,9}$. Ademais, os movimentos de mastigação iniciamse efetivamente em torno de 6 meses, época na qual é recomendada a introdução de alimentos mais espessos ${ }^{3}$.

São freqüentes os distúrbios gastrintestinais, particularmente a diarréia, em lactentes que recebem precocemente alimentos com amido ${ }^{3}$. Por sua vez, a introdução precoce de outros alimentos, como os cereais e vegetais, na dieta de crianças amamentadas pode comprometer a absorção de ferro por mecanismos de quelação ${ }^{7}$, e o excesso de amido não digerido pode interferir na absorção de outros nutrientes e resultar em falha de crescimento ${ }^{3}$. Alimentos preparados também têm maior chance de contaminação, determinando diarréias infecciosas.

A desnutrição pode estar relacionada a práticas inadequadas de desmame, e a qualidade dos alimentos consumidos pelas crianças pode refletir-se, a curto e longo prazo, na saúde infantil, por uma programação nutricional que explicaria doenças da idade adulta relacionadas a práticas nutricionais inadequadas no período neonatal ${ }^{21}$ e na infân$\mathrm{cia}^{22}$. Tomando como exemplo a obesidade, as crianças amamentadas têm melhor capacidade de manipular os níveis séricos de colesterol do que as que recebem fórmulas lácteas, com indicativos de prevenção da obesidade na idade adulta 22 .

Na população estudada, notou-se uma associação entre o uso de chupeta e o desmame precoce. Diversos estudos têm mostrado uma associação inversa entre o uso de chupeta e o aleitamento materno ${ }^{23-25}$. Um possível mecanismo seria a redução da freqüência das mamadas diárias, o que poderia levar à baixa estimulação da mama e à conseqüente diminuição da produção de leite ${ }^{26}$. No entanto, ainda não ficou bem estabelecida uma relação causal. Recente estudo sugere que o uso de chupeta deve ser visto como um indicador de problema com a amamentação, porque as mães introduziram a chupeta quando demonstraram alguma dificuldade com a amamentação 27 .

A escolaridade materna e a renda familiar sempre figuram como determinantes nos estudos epidemiológicos. No entanto, neste estudo, não foram observadas diferenças estatisticamente significativas quando se avaliou a introdução de refeição da família na alimentação das crianças menores ou iguais a 4 meses segundo a presença dessas variáveis. Esse fato sugere que os conhecimentos sobre as práticas alimentares, em Feira de Santana, estão relacionados com referenciais que permeiam diferentes estratos sociais, evidenciando a necessidade do desenvolvimento de estudos que possibilitem ampliar a compreensão acerca dos valores culturais do município.

Por fim, a análise dos alimentos líquidos e semi-sólidos/ sólidos consumidos pelas crianças amamentadas e nãoamamentadas revelou que a amamentação esteve associada a um melhor comportamento alimentar, evidenciado pela menor prevalência de consumo de alimentos semisólidos/sólidos antes dos 4 meses de vida.

Foi constatado também que o consumo de suco e água pelas crianças amamentadas interfere menos do que o chá nos indicadores de aleitamento exclusivo, sinalizando a necessidade de medidas de intervenção através de um trabalho educativo com a população. Sabe-se que mudar hábitos culturalmente estabelecidos é difícil, e é necessário um trabalho contínuo, que respeite as características da comunidade. No processo de mudança, é preciso conscientizar os profissionais de saúde e os indivíduos formadores de opinião e estimular programas de educação para orientar as gestantes e mães sobre a alimentação da criança no primeiro ano de vida. 
É importante lembrar que o sucesso das práticas alimentares do lactente depende de suprir a criança com alimentos de adequada qualidade, que satisfaçam as necessidades nutricionais, protejam contra a absorção de substâncias alimentares estranhas, não excedam a capacidade funcional do trato gastrintestinal e estejam livres de agentes infecciosos; todas essas características estão presentes no leite humano.

\section{Referências}

1. World Health Organization. The optimal duration of exclusive breastfeeding: a systematic review. Geneva: WHO; 2001.

2. World Health Organization. Report of the expert consultation on the optimal duration of exclusive breastfeeding. Geneva: WHO; 2001.

3. World Health Organization. Complementary feeding of young children in developing countries: a review of current scientific knowledge. Geneva: WHO; 1998.

4. Giugliani ERJ, Victora CG. Alimentação Complementar. J Pediatr (Rio J). 2000;76(Suppl 3):253-62.

5. Rea MF. O pediatra e a amamentação exclusiva. J Pediatr (Rio J). 2003;79:479-80.

6. Vieira GO, Almeida JAG. Leite materno como fator de proteção contra doenças do tubo digestivo. In: Silva LR, organizadora. Urgências clínicas e cirúrgicas em gastroenterologia e hepatologia pediátricas. Rio de Janeiro: MEDSI; 2004. p. 951-9.

7. Vieira GO. Alimentação infantil e morbidade por diarréia na cidade de Feira de Santana, 2001 [dissertação]. Feira de Santana (BA): Universidade Estadual de Feira de Santana; 2002.

8. Ramos M, Stein LM. Desenvolvimento do comportamento alimentar infantil. J Pediatr (Rio J). 2000;76(Suppl 3):228-37.

9. Euclydes MP. Nutrição do lactente: base científica para uma alimentação adequada. $2^{\text {a }}$ ed. Minas Gerais: Suprema; 2000

10. Giugliani ERJ, Lopez FA. Uma atualização em nutrição infantil. J Pediatr (Rio J). 2000;76(Suppl 3):227-8.

11. Golan M. Influencia dos fatores ambientais domésticos no desenvolvimento e tratamento da obesidade infantil. Anais Nestlé 2002;62:31-42.

12. Ministério da Saúde. Prevalência do aleitamento materno nas capitais brasileiras e no Distrito Federal. Brasília: Ministério da Saúde/Secretária de Políticas de Saúde/Área de Saúde da Criança; 2001.

13. Montrone CVG, Arantes IS. Prevalência do aleitamento materno na cidade de São Carlos, São Paulo. J Pediatr (Rio J). 2000;76:138-42.

14. Moura EFA. Duração do período de aleitamento materno de crianças atendidas em ambulatório de pediatria. J Pediatr (Rio J). $1997 ; 73: 106-10$.
15. Caldeira AP, Goulart EMA. A situação do aleitamento em Montes Claros, Minas Gerais: estudo de uma amostra representativa. J Pediatr (Rio J). 2000;76:65-71.

16. Lamounier JA. Tendências do aleitamento materno no Brasil. Rev Med Minas Gerais. 1999;9:59-65.

17. World Health Organization. Collaborative Study Team on the role of breastfeeding on the prevention of infant mortality. Effect of breastfeeding on infant and child mortality due to infectious diseases in less developed countries: a pooled analysis. Lancet. 2000;355:451-5.

18. Cesar JA, Victora CG, Barros FC, Santos IS, Flores JA. Impact of breastfeeding on admission for pneumonia during postneonatal period in Brazil: nested case-control study. BMJ. 1999;318: 1316-20.

19. Arifeen S, Black RE, Antelman G, Baqui A, Caulfield L, Becker S. Exclusive breastfeeding reduces acute respiratory infection and diarrhea deaths among infants in Dhaka Slums. Pediatrics. 2001;108:167-71.

20. Ministério da Saúde. Pesquisa Nacional de Demografia e Saúde 1996 - PNDS/96. Brasília: Sociedade Civil do Bem-estar Familiar no Brasil - BEMFAM; 1997.

21. Anderson JW, Johnstone BM, Remly DT. Breast-feeding and cognitive development: a meta-analysis. Am J Clin Nutr. 1999; 70:525-35.

22. Oliveira FLC, Escrivão MAMS. Prevenção na infância e adolescência das doenças do adulto. In: Temas de nutrição em pediatria. Rio de Janeiro: Departamento de Nutrição/Sociedade Brasileira de Pediatria; 2001.

23. Soares MEM, Giugliani ERJ, Braun ML, Salgado ACN, Oliveira AP, Aguiar PR. Uso de chupetas e sua relação com o desmame precoce em população de crianças nascidas em Hospital Amigo da Criança. J Pediatr (Rio J). 2003;79:309-16.

24. Gorbe E, Kohalmi B, Gaal G, Szantho A, Rigo J, Harmath A, Csabay $L$, et al. The relationship between pacifier use, bottle feeding and breast feeding. J Matern Fetal Neonatal Méd. 2002;12:127-31.

25. Howard CR, Howard FM, Lanphear B, Eberly S, DeBlieck EA Oakes $D$, et al. Randomized clinical trial of pacifier use and bottle-feeding or cupfeeding and their effect on breastfeeding. Pediatrics. 2003;11:511-8.

26. Lamounier JA. O efeito de bicos e chupetas no aleitamento materno. J Pediatr (Rio J). 2003;79:284-6.

27. Coutinho SB. Aleitamento materno exclusivo: um estudo de intervenção randomizado na zona da mata meridional de Pernambuco [tese]. Recife (PE): Universidade Federal de Pernambuco; 2003.

\section{Correspondência:}

Graciete Oliveira Vieira

Rua Barão Rio Branco, 1499 - Centro

CEP 44025-000 - Feira de Santana, BA

Fones: (75) 221.3884/625.4156

Fax: (75) 223.2351

E-mail: graciete.vieira@terra.com.br 